
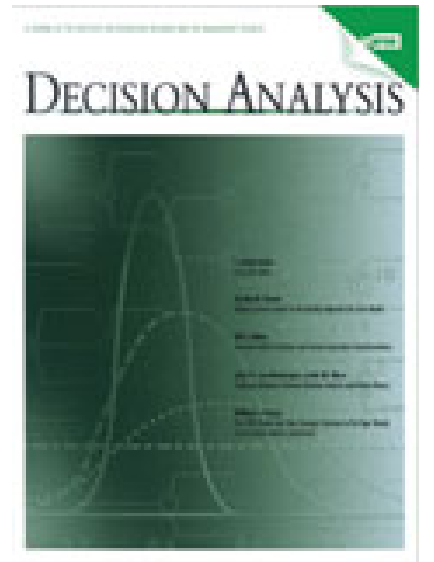

\title{
Decision Analysis
}

Publication details, including instructions for authors and subscription information:

http:// pubsonline.informs.org

\section{Augmented Markov Chain Monte Carlo Simulation for Two- Stage Stochastic Programs with Recourse}

Tahir Ekin, Nicholas G. Polson, Refik Soyer

\section{To cite this article:}

Tahir Ekin, Nicholas G. Polson, Refik Soyer (2014) Augmented Markov Chain Monte Carlo Simulation for Two-Stage Stochastic Programs with Recourse. Decision Analysis 11(4):250-264. http:// dx. doi. org/ 10.1287/ deca. 2014.0303

\section{Full terms and conditions of use: http://pubsonline.informs.org/page/terms-and-conditions}

This article may be used only for the purposes of research, teaching, and/or private study. Commercial use or systematic downloading (by robots or other automatic processes) is prohibited without explicit Publisher approval, unless otherwise noted. For more information, contact permissions@informs.org.

The Publisher does not warrant or guarantee the article's accuracy, completeness, merchantability, fitness for a particular purpose, or non-infringement. Descriptions of, or references to, products or publications, or inclusion of an advertisement in this article, neither constitutes nor implies a guarantee, endorsement, or support of claims made of that product, publication, or service.

Copyright @ 2014, INFORMS

Please scroll down for article-it is on subsequent pages

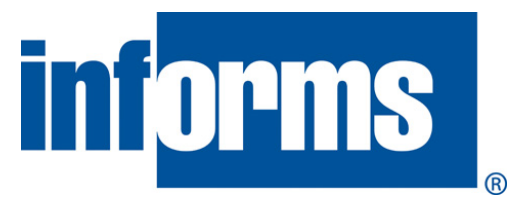

INFORMS is the largest professional society in the world for professionals in the fields of operations research, management science, and analytics.

For more information on INFORMS, its publications, membership, or meetings visit http:// www. informs. org 


\title{
Augmented Markov Chain Monte Carlo Simulation for Two-Stage Stochastic Programs with Recourse
}

\author{
Tahir Ekin \\ McCoy College of Business, Texas State University, San Marcos, Texas 78666, t_e18@txstate.edu \\ Nicholas G. Polson \\ Booth School of Business, University of Chicago, Chicago, Illinois 60637, ngp@chicagobooth.edu \\ Refik Soyer \\ School of Business, George Washington University, Washington, DC 20052, soyer@gwu.edu
}

\begin{abstract}
Tn this paper, we develop a simulation-based approach for two-stage stochastic programs with recourse. We construct an augmented probability model with stochastic shocks and decision variables. Simulating from the augmented probability model solves for the expected recourse function and the optimal first-stage decision. Markov chain Monte Carlo methods, together with ergodic averaging, provide a framework to compute the optimal solution. We illustrate our methodology via the two-stage newsvendor problem with unimodal and bimodal continuous uncertainty. Finally, we present performance comparisons of our algorithm and the sample average approximation method.

Keywords: decision analysis; dynamic decision making; math programming; optimization; Markov chain Monte Carlo

History: Received on March 7, 2013. Accepted by Editor-in-Chief Rakesh K. Sarin on August 13, 2014, after 3 revisions. Published online in Articles in Advance September 22, 2014.
\end{abstract}

\section{Introduction}

Stochastic programming with recourse provides a number of computational challenges for a sequential decision maker; see Dantzig (1955). This class of decision problems is characterized by the possibility of a corrective recourse action after resolution of uncertainty. Our paper develops a simulation-based approach for computing the first- and second-stage optimal decisions. To do this, we define an augmented probability model over the sources of stochastic uncertainty and the decision variables. We design a Markov chain Monte Carlo (MCMC) algorithm with a corresponding marginal distribution on the decision that collapses on the optimal first-stage decision. Our approach builds on previous literature; see Bielza et al. (1999), Müller et al. (2004), and Jacquier et al. (2007).

We focus on two-stage problems with recourse. A stage corresponds to a point of time where a set of decision variables is chosen. The decision maker computes an optimal first-stage decision under uncertainty and a second-stage decision after observing stochastic uncertainties. The first-stage, or "here-and-now," decision is denoted by $x$. The stochastic uncertainty, $\xi$, is drawn from a known probability distribution $p(\xi)$, which is flexible. Optimal decisions are made by maximizing the continuation expected utility. The optimal first-stage decision is feasible for all scenarios of the random variable $\xi$ so that the optimal expected reward (or penalty) associated with both stages is realized. After uncertainty is revealed, the second-stage decision, $y(x, \xi)$, is determined by optimizing the second-stage problem for the specific combination of first-stage decision and the outcome value $(x, \xi)$. To solve this, we have the following dynamic program, where $Q(x, \xi)$ is the recourse function:

$$
\max _{x}\left\{m x-E_{\xi}[Q(x, \xi)]\right\}
$$

subject to $A x \leqslant b, x \geqslant 0$,

$$
\begin{array}{r}
\text { where } Q(x, \xi)=\min _{y} q(\xi) y \\
\text { subject to } T x+W y \geqslant h, y \geqslant 0 .
\end{array}
$$

At the first stage, we compute $\max _{x}$ rather than $\min _{x}$ in the standard formulation. This notation will help us to 
Figure 1 Decision Tree Representation of the Two-Stage Recourse Problem

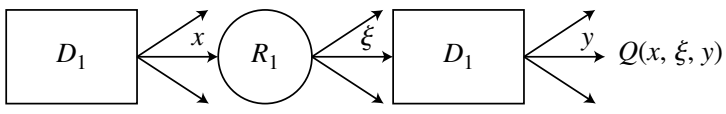

define our augmented probability model. The secondstage decision, $y(x, \xi)$, depends on the initial decision $x$ and the source of uncertainty $\xi$. The recourse function $Q(x, \xi)$ involves the minimization of $(q(\xi) y)$ subject to the second-stage constraints. Here, $q(\xi)$, the coefficient of the second-stage objective function, is a function of the random variable $\xi$, and it is assumed to be integrable; $E[|q(\xi)|]<\infty$. Although $h$ is a deterministic parameter, this formulation can be extended to allow $h$ to be a function of a random variable. The matrices $W$ and $T$ have appropriate dimensions conformable with $x$ and $y$. The parameters $m, A$, and $b$ are prespecified, deterministic arrays with appropriate dimensions.

Figure 1 illustrates the sequential nature of events. The solution involves "folding back" the decision tree while satisfying the first- and second-stage constraints. Specifically, if we assume $Q(x, \xi, y)=m x-q(\xi) y$, then the solution at the second decision node is given by $Q(x, \xi)=\min _{y} q(\xi) y$, given that $y$ satisfies the secondstage constraints. Taking the expectation with respect to the probability distribution of $\xi, p(\xi)$, at the first random node and maximizing with respect to $x$ at first decision node yields the full formulation.

\subsection{Newsvendor Problem}

To illustrate our methodology, we consider the classic two-stage stochastic program with recourse known as the newsvendor problem. See Dai et al. (2000), Parpas et al. (2013), and Homem-de-Mello and Bayraksan (2014) for the two-stage stochastic programming setup of the problem. Suppose that a newsvendor can choose the number of newspapers, $x$, to be purchased at the first stage. We assume that $\boldsymbol{\xi}=\left(\xi_{1}, \xi_{2}\right)$ represent uncertainty with demand, $d\left(\xi_{1}\right)$, and unit sales price, $s\left(\xi_{2}\right)$. The functions $d\left(\xi_{1}\right)$ and $s\left(\xi_{2}\right)$ are assumed to be nonnegative. We denote the unit purchase price of a newspaper at the first stage by $c$ and the unit resale (salvage) price at the second stage by $r$ such that $c \geqslant r$. The second-stage decisions on quantities of newspapers sold and salvaged are denoted by $y_{1}$ and $y_{2}$, respectively. The newsvendor's problem is to determine $x$ so that the overall expected profit is maximized. The problem can then be formulated as

$$
\max _{x}\left\{-c x+E_{\xi}[Q(x, \xi)]\right\} \quad \text { such that } x \geq 0,
$$

with $m=-c$. The recourse function, $Q(x, \boldsymbol{\xi})$, is determined by the linear program

$$
Q(x, \xi)=\max _{y_{1}, y_{2}}\left\{s\left(\xi_{2}\right) y_{1}+r y_{2}\right\}
$$

subject to $y_{1} \leq d\left(\xi_{1}\right), y_{1}+y_{2} \leq x, y_{1} \geq 0, y_{2} \geq 0$.

Once the uncertainty, $\xi$, is revealed, the outcomes of $d\left(\xi_{1}\right)$ and $s\left(\xi_{2}\right)$ are realized, and the optimal recourse action from the linear program is given by

$$
\begin{aligned}
& y_{1}^{*}(x, \boldsymbol{\xi})=\min \left(d\left(\xi_{1}\right), x\right) \text { and } \\
& y_{2}^{*}(x, \boldsymbol{\xi})=\max \left(x-d\left(\xi_{1}\right), 0\right)
\end{aligned}
$$

for $s\left(\xi_{2}\right) \geqslant r$ and

$$
y_{1}^{*}(x, \boldsymbol{\xi})=0 \text { and } y_{2}^{*}(x, \boldsymbol{\xi})=x
$$

for $s\left(\xi_{2}\right) \leqslant r$. The goal then is to solve the newsvendor problem with our MCMC-based methodology.

The rest of the paper is outlined as follows. Section 2 defines our notation and provides a literature review. Section 3 defines our augmented probability model for stochastic programs that we use to simulate the optimal decisions and presents an MCMC algorithm using a stylized example and the newsvendor problem. Section 4 provides implementation of the augmented probability simulation (APS) model to solve the newsvendor problem for unimodal and bimodal demand distributions and compares the performance of our approach with the sample average approximation method. Finally, $\S 5$ concludes with directions for further research.

\section{Simulation-Based Stochastic Programming}

The main computational difficulty in solving a twostage problem with recourse is presented by the evaluation of the expected recourse function $E_{\xi}[Q(x, \xi)]$, where $Q(x, \xi)$ is the optimal value function for the problem $\min _{y} q(\xi) y$, subject to constraints $(T x+W y \geqslant h$ and 
$y \geqslant 0)$. When the stochastic uncertainty follows a discrete distribution with a small number of scenarios, the problem can be rewritten deterministically and solved using the simplex or interior point methods. A common approach for this is Benders' algorithm (see Benders 1962, Van Slyke and Wets 1969), which uses a planecutting technique also known as the L-shaped method. With a discrete source of uncertainty, this has the advantage of scalability. The basic idea is to represent the second-stage problem by a scalar variable that cuts the objective function. The scalar variable provides an outer approximation to the expected recourse function, and the cuts represent the second-stage constraints in terms of first-stage decision variable. A variant is the multicut L-shaped algorithm of Birge and Louveaux (1988).

More generally, computation becomes more difficult for continuous sources of uncertainty. Successive discrete approximation methods such as computing Jensen inequalities and Edmundson-Madansky bounds using quadrature rules (Pennanen and Koivu 2005) have been proposed to solve these problems, as have Monte Carlo methods. Homem-de-Mello and Bayraksan (2014) provide an extensive survey of simulation-based methods.

One advantage of the Monte Carlo methods is that they are computationally tractable and applicable to both discrete and continuous sources of uncertainty. To implement direct Monte Carlo, we draw $\xi^{(g)}$ from $p(\xi), G$ times. Given this set of $G$ sampled scenarios, we approximate the expected recourse function $E_{\xi}[Q(x, \xi)]=\int Q(x, \xi) P(d \xi)$ via $\hat{E}_{\xi}[Q(x, \xi)]=$ $(1 / G) \sum_{g=1}^{G} Q\left(x, \xi^{(g)}\right)$.

Convergence in $G$ is guaranteed by the law of large numbers, $\hat{E}_{\xi}[Q(x, \xi)] \rightarrow E_{\xi}[Q(x, \xi)]$, as $G \rightarrow \infty$ provided that $E_{\xi}[|Q(x, \xi)|]<\infty$; see, for example, Dempster et al. (1977). Given this estimate of the expected recourse function, the so-called master or sample average approximation (SAA) problem is solved as a deterministic program:

$$
\max _{x}\left\{m x-\frac{1}{G} \sum_{g=1}^{G} Q\left(x, \xi^{(g)}\right)\right\}
$$

subject to $A x \leqslant b, x \geqslant 0$,

$$
\text { where } Q\left(x, \xi^{(g)}\right)=\min _{y} q\left(\xi^{(g)}\right) y
$$

subject to $T x+W y \geqslant h, y \geqslant 0$.
Direct Monte Carlo methods have the advantage that they are straightforward to implement, but they can be computationally expensive in high dimensions. Various variance reduction techniques such as antithetic variates, Latin hypercube sampling, quasi-Monte Carlo methods, and control variates can also be used. Other alternatives are importance sampling methods, where rather than directly drawing from $p(\xi)$, one uses an importance proposal distribution, $g(\xi)$, within the L-shaped method. The proposal distribution is designed to place more weight in the region of high $Q(x, \xi)$ values, thus providing a more efficient estimate of $E_{\xi}[Q(x, \xi)]$. Proposals for $g(\xi)$ are described as additive (Infanger 1993) or multiplicative (Dantzig and Thapa 1997) functions.

Sequential or internal sampling solution methods, where sampling is embedded within the optimization approach, have also been proposed. This strategy aims to avoid the cost of the sampling effort when the current iterative solution is far from the optimal. The stochastic decomposition (SD) method of Higle and Sen (1991, 1996) uses direct Monte Carlo methods to estimate the expectation functions rather than computing them exactly as in regular L-shaped methods. The cuts are also updated within the algorithm as additional sample points are drawn while the algorithm proceeds. One difficulty with this approach is that the convergence to an optimum may only occur on a subsequence and that the existing cuts become redundant in the later stages of the algorithm. Regularized SD algorithms attempt to eliminate the excess cuts by adding a regularizing term as in Higle and Sen (1996). Spall (2005) provides an extensive review of the stochastic approximation algorithms that are initially considered by Rubinstein and Shapiro (1993). Ermoliev (1988) and Gaivoronski (1988) discuss the class of stochastic quasigradient methods, which are stochastic versions of subgradient methods designed for optimizing convex objective functions. Although all these methods can be effective in low-dimensional problems, they can have poor convergence properties in high dimensions because of possible nonoptimal step sizes (Spall 2005).

The fundamental issue in any simulation-based optimization approach is the assessment of quality of the solution and asymptotic properties of the sample estimates. If the optimal solution and objective function values are analytically available via integration, we can 
evaluate the quality of the solution in a straightforward manner. The difficulty arises in evaluating expectation functions analytically for many stochastic programs with continuous uncertainty; assessment of the quality of the proposed solution is not trivial, and many approaches have been proposed. For example, Higle and Sen (1996) discuss stopping criteria based on duality and optimality conditions. Shapiro and Homemde-Mello (1998) develop statistical inference for the estimation of error and propose statistically based stopping criteria for iterative algorithms. Bayraksan and Morton (2009) provide a detailed discussion and comparison of different procedures. In this paper, we use the multiple replications procedure (MRP) that is proposed by Mak et al. (1999) and revisited by Bayraksan and Morton (2009). Shapiro (2003) and Shapiro et al. (2009) discuss the theoretical properties of sampling-based approaches comprehensively. Dai et al. (2000), Shapiro and Homem-de-Mello (2000), and Polson and Sorensen (2011) also provide discussions and comparisons of the convergence rates of simulationbased optimization methods.

\section{An Augmented Probability Simulation Approach}

In this section we develop an augmented probability model for solving the two-stage program (1) by maximizing the objective function

$$
m x-E_{\xi}[Q(x, \xi)]
$$

with respect to $x$, where $Q(x, \xi)=\min _{y(x, \xi)} q(\xi) y(x, \xi)$ and $(x, y(x, \xi))$ satisfy the constraints $\{A x \leqslant b, x \geqslant 0$, $T x+W y \geqslant h, y \geqslant 0\}$. In many cases such as the newsvendor problem, the optimal recourse action, $y^{*}(x, \xi)$, is available in closed form, which in turn provides $Q(x, \xi)=q(\xi) y^{*}(x, \xi)$. In such cases, finding the firststage solution $x$ reduces to maximizing the expected value of

$$
u(x, \xi)=m x-q(\xi) y^{*}(x, \xi)
$$

while satisfying the first-stage constraints $\{A x \leqslant b$, $x \geqslant 0\}$.

As proposed by Bielza et al. (1999), Müller (1999), and Müller et al. (2004), maximization of the expected value of $u(x, \xi)$ can be achieved by simulation from an augmented probability distribution $\pi(x, \xi)$ of the decision variable $x$ and uncertainty shock $\xi$. Specifically, define an auxiliary distribution

$$
\pi(x, \xi)=\frac{u(x, \xi) p(\xi)}{Z}
$$

where $Z=\int u(x, \xi) p(\xi) \mathrm{d} x \mathrm{~d} \xi$ is an appropriate normalization constant. This leads to a marginal distribution of $x$ given by

$$
\pi(x)=\frac{1}{Z} \int u(x, \xi) p(\xi) \mathrm{d} \xi,
$$

which is proportional to the expected value of $u(x, \xi)$. Then the optimal value of first-stage decision $x$ is given by the mode of the marginal distribution $\pi(x)$. In the above, we assume that $u(x, \xi)$ is a nonnegative function; that is, $m x-q(\xi) y^{*}(x, \xi) \geqslant 0$. (See Jacquier et al. 2010 for transformations such as adding a positive constant to satisfy this assumption.)

We simulate from the augmented probability model $\pi(x, \xi)$ using MCMC. Specifically, we use a Gibbs sampler. This will require simulation from the conditional distribution $\pi(\xi \mid x)$, which is a "tilted" version of the distribution $p(\xi)$ and depends on $y^{*}(x, \xi)$, the solution to the optimal second-stage recourse function. The conditional distribution is given by $\pi(\xi \mid x) \propto u(x, \xi) p(\xi)$, where $\propto$ implies that the probability density is defined up to a constant of proportionality. We also need to be able to simulate from the conditional distribution $\pi(x \mid \xi) \propto u(x, \xi)$.

Our approach is more efficient than direct Monte Carlo (MC), which involves first approximating $E_{\xi}[Q(x, \xi)]$ by $\hat{E}_{\xi}[Q(x, \xi)]=(1 / G) \sum_{g=1}^{G} Q\left(x, \xi^{(g)}\right)$ using $G$ independent MC samples from $p(\xi)$ and then optimizing $\left(m x-\hat{E}_{\xi}[Q(x, \xi)]\right)$ over $x$. This is inefficient in high dimensions because the MC errors in the approximation $\hat{E}_{\xi}[Q(x, \xi)]$ can overwhelm the calculation of the optimal $x$.

The augmented probability simulation approach can be extended to include the second-stage decision functions, $y(x, \xi)$, and our approach can also be used to obtain this optimal decision. In such cases, we then define the augmented joint distribution as $\pi(x, \xi, y)$, and sampling from the conditional distribution $\pi(y \mid$ $x, \xi)$ will be required. To do that, the algorithm of Pincus (1968) is utilized. The resulting Algorithm 2 is presented in §3.1.4. 
3.1. Algorithm for Two-Stage Stochastic Programs Consider the optimization problem $\min _{x}\{Q(x) \mathbb{I}(A x \geqslant$ $b, x \geqslant 0)\}$, where $Q(x)$ is continuous and attains a global unique minimum, and $\mathbb{1}(\cdot)$ denotes an indicator function. Define a joint distribution where $J$ denotes an algorithmic augmentation parameter

$$
\pi_{J}(x) \triangleq \frac{1}{Z_{J}} \exp \{-J Q(x)\} \mathbb{1}(A x \geqslant b, x \geqslant 0) .
$$

Here, $Z_{J}=\int \exp \{-J Q(x)\} \mathrm{d} x \mathbb{I}(A x \geqslant b, x \geqslant 0)$ is the normalization constant. The following simulation property was initially proposed by Pincus (1968): the mode of the distribution, $\pi_{J}(x)$, is equal to the optimal decision, $x^{*}=\arg \min _{x}\{Q(x) \mathbb{I}(A x \geqslant b, x \geqslant 0)\}$, and as $J \rightarrow \infty$, the mean, $E\left[\pi_{J}(x)\right] \rightarrow x^{*}$, converges to the desired mode.

For a fixed value of $J$, Pincus (1970) proposes a Metropolis algorithm to simulate $\left(x^{(0)}, x^{(1)}, \ldots, x^{(g)}\right.$, $\left.\ldots, x^{(G)}\right)$ with the equilibrium distribution, $\pi_{J}(x)$. Under mild Harris recurrence conditions, given any starting point $x^{(0)}$, we have the limiting property $\lim _{G \rightarrow \infty} \mathbb{P}\left(x^{(G)} \in A \mid x^{(0)}=x\right)=\pi_{J}(A)$ for any Borel set $A$. See Tierney (1994), who ensures MCMC convergence in a number of iterations, $G$, for $\pi_{J}(x)$ with a fixed value of $J$. Hence, if we can perform an MCMC simulation of $\pi_{J}(x)$, we can calculate the mean as an estimator of $x^{*}$. We now provide theoretical convergence properties of our MCMC algorithm, which adopts $J=J_{n}, n=1,2, \ldots$, where $J_{n} \rightarrow \infty$ at an appropriate rate to guarantee convergence to the mode.

3.1.1. Convergence Properties. The limiting cases where $J=0$ or $\infty$ are of particular interest. They both lead to uniform measures but on different sets: $J=0$ leads to a uniform measure on the constraint set $\mathscr{X}$, and $J=\infty$ leads to a uniform measure on the set of modes $\mathscr{X}_{\min }$. Specifically, with $\delta$ denoting a Dirac measure, we have

$$
\lim _{J \rightarrow \infty} \pi_{J}(x)=\pi_{\infty}(x)=\left|\mathscr{X}_{\text {min }}\right|^{-1} \delta_{\mathscr{X}_{\text {min }}}(x) ;
$$

see Geman and Geman (1987). We now let $J=J_{n}$, $n=1,2, \ldots, \infty$, vary according to a cooling schedule. This creates a time-inhomogeneous chain (see Isaacson and Madsen 1976) of draws $\left(x^{(g)}, J^{(g)}\right)$ that converges to $\pi_{\infty}(x)$ and hence finds the optimum $x^{\star}$. For changing values of $n$, one obtains an inhomogeneous chain since the target density changes with $n$. Increasing $J_{n}$ too fast would possibly cause it to get stuck in a local mode. General convergence properties of an inhomogeneous Markov chain follow from Müller et al. (2004).
Our augmented probability model is defined by using $J$ independent and identically distributed (iid) draws of $\xi, \boldsymbol{\xi}_{J}=\left(\xi_{1}, \ldots, \xi_{J}\right)$, where we assume bounded utility (objective) functions; $u_{0} \leqslant u\left(x, \xi_{j}\right) \leqslant u_{1}$ for all $J$. The distribution of interest, $\pi_{J}(x)$, is the marginal distribution implied from the joint distribution

$$
\pi_{J}\left(x, \boldsymbol{\xi}_{J}\right) \propto \prod_{j=1}^{J}\left[u\left(x, \xi_{j}\right) p\left(\xi_{j}\right)\right] .
$$

Equivalently, let $v=(1 / J) \sum_{j=1}^{J} \log u\left(x, \xi_{j}\right)$ be the average of $\log$ utility functions. Define the transition probabilities: $P_{i j}(n)=P\left(x_{n+1}=x^{\prime}, v_{n+1}=v^{\prime} \mid x_{n}=x, v_{n}=v\right)$ on the space of $(x, v)$ for $i=(x, v)$ and $j=\left(x^{\prime}, v^{\prime}\right)$ with the stationary distribution $\pi_{J}^{*}(x, v)$. We assume there exist constants $0<a<\infty$ and $0 \leqslant b<\infty$, and we assume that $p(v \mid x) \geqslant \epsilon>0$ for all $(x, v)$, so that $p_{J}(v \mid x) \geqslant \epsilon^{J}$ and that the proposal density $p\left(x^{\prime} \mid x\right) \geqslant a$. This allows us to have a bound on the transition kernel, $P_{i j}(n)$, which is related to the coefficient of ergodicity:

$$
\tau[P(n)]=1-\inf _{i, j} \sum_{l=1}^{m} \inf \left(P_{i l}(n), P_{j l}(n)\right) .
$$

The convergence rate is characterized by

$$
\delta[P(n)]=1-\tau[P(n)],
$$

which is referred to as the delta coefficient of $P(n)$; see Isaacson and Madsen (1976).

As pointed out by Müller et al. (2004), to show weak ergodicity to the desired stationary distribution, $\pi_{\infty}^{*}(x, v)$, we pick $J_{n}$ to ensure that $\sum_{n=0}^{\infty}\{1-\tau[P(n)]\}=\infty$ for our sequence of stationary distributions. For a fixed $J_{n}$, a bound is also established, $P_{i j}(n) \geqslant a \exp \left\{-b J_{n}\right\}$, that results with geometric convergence with a rate characterized by $\delta[P(n)]$. By setting $b=\log \left(u_{1} /\left(u_{0} \epsilon\right)\right)$ and using the assumptions on the utility, we have

$$
P_{i j}(n) \geqslant a\left(u_{0} \epsilon / u_{1}\right)^{J_{n}}=a \exp \left\{-b J_{n}\right\} .
$$

If we pick the cooling schedule, $J_{n}=(1 / \gamma) \log (n+c)$, then

$$
\sum_{n=0}^{\infty}[1-\tau(P(n))] \geqslant a \sum_{n=0}^{\infty} \exp \left\{-b J_{n}\right\}=a \sum_{n=0}^{\infty} 1 /(n+c)^{b / \gamma}=\infty,
$$

which holds for $c>0$ and $\gamma \geqslant b$. Therefore we have weak ergodicity of our chain. Strong ergodicity follows from the Theorem A.1 of Müller et al. (2004). 
For practical purposes, instead of a formal implementation, we rather recommend a diagnostic approach that increases $J^{(g)}$ until the MCMC draws stabilize (see Gelman and Rubin 1992). The geometric rate of convergence depends on $b$, a function of the bounds of the utility, $u_{0}$ and $u_{1}$. Practically speaking, these bounds can be chosen with respect to the maximum and minimum values of the utility function, $\max Q(x)$ and $\min Q(x)$, respectively. It is then enough to sample from the joint density and calculate the ergodic mean $(1 / G) \sum_{g=1}^{G} X^{(g)}$ along the chain for a value of $J$ on a given schedule. Figure 2 presents evidence for numerical convergence rate that is geometric for increasing values of $J$. Practical convergence in $J$ is also assessed for reasonable values of $J$ such as 5, 10, 25, 50, or 100 in most applications (Jacquier et al. 2007). The Brooks-Gelman-Rubin (BGR) statistic, which is an analysis-of-variance-type diagnostic that compares within- and among-chain variance (Brooks and Roberts 1998), is used as the practical MCMC convergence diagnostic in this paper. BGR values around 1 indicate convergence.

3.1.2. Modified Augmented Probability Model. Given these convergence results, we modify our APS by defining the augmented probability model with $J$ iid draws of $\xi$ as

$$
\begin{gathered}
\pi_{J}\left(x, \boldsymbol{\xi}_{J}\right) \propto \prod_{j=1}^{J}\left[\left(m x-q\left(\xi_{j}\right) y_{j}\right) p\left(\xi_{j}\right) \mathbb{I}(A x \leqslant b, x \geqslant 0,\right. \\
\left.\left.T x+W y_{j} \geqslant h, y_{j} \geqslant 0\right)\right],
\end{gathered}
$$

where for each $x, J$ unique draws of $\left(\xi_{j}\right)$ are generated and $y_{j}^{*}=y^{*}\left(x, \xi_{j}\right)$ are computed for each pair. We

\section{Figure 2 (Color online) Optimality Gaps for Different Values of $J$}

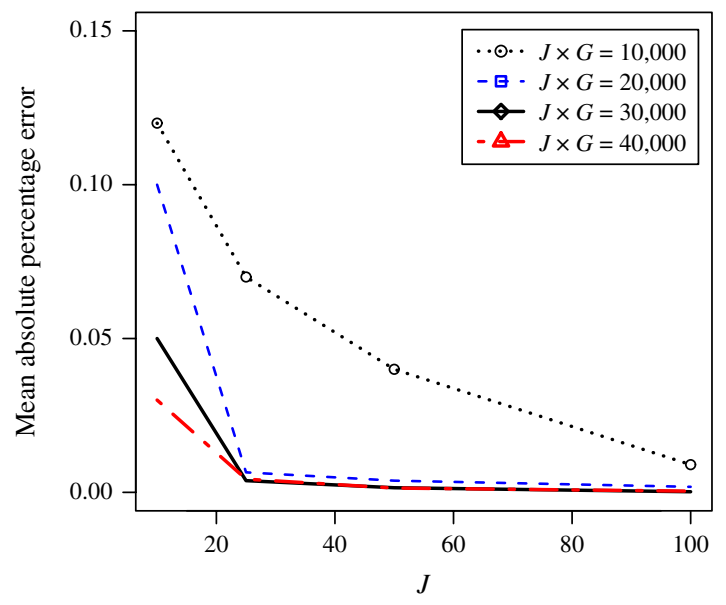

assume that $\left(m x-q\left(\xi_{j}\right) y_{j}^{*}\right) \geqslant 0$ for all $J$ terms, and $\boldsymbol{\xi}_{J}=\left(\xi_{1}, \ldots, \xi_{J}\right)$ are $J$ independent draws from $p(\xi)$.

The key feature of this distribution is that the mean of its marginal distribution $\pi_{J}(x)$ for the first-stage variable converges to the optimum for a sufficiently large enough value of $J$. Since $\xi_{j}$ 's are iid draws, the marginal distribution is given by

$$
\begin{array}{r}
\pi_{J}(x) \propto E_{\xi}[(m x-q(\xi) y) \mathbb{1}(A x \leqslant b, x \geqslant 0, \\
T x+W y \geqslant h, y \geqslant 0)]^{J},
\end{array}
$$

where

$$
E_{\xi}[(m x-q(\xi) y)]^{J}=\left[\int(m x-q(\xi) y) p(\xi) \mathrm{d} \xi\right]^{J} .
$$

This corresponds to our previous discussion. The marginal distribution $\pi_{J}(x) \propto e^{Q(x)}$, where

$$
\begin{array}{r}
Q(x)=\ln E_{\xi}[(m x-q(\xi) y) \mathbb{1}(A x \leqslant b, x \geqslant 0, \\
T x+W y \geqslant h, y \geqslant 0)]^{J} .
\end{array}
$$

Therefore by computing the mean of the samples from $\pi_{I}(x)$, we can find the optimal first-stage decision $x^{*}$.

To sample from $\pi_{J}\left(x, \boldsymbol{\xi}_{J}\right)$, we use MCMC. A Gibbs sampler can be constructed using conditional distributions $\pi_{J}\left(x \mid \boldsymbol{\xi}_{J}\right)$ and $\pi_{J}\left(\boldsymbol{\xi}_{J} \mid x\right)$. Samples from the joint conditional distribution $\pi_{J}\left(\xi_{J} \mid x\right)$ can be obtained by independent draws from the conditionals $\pi_{J}\left(\xi_{j} \mid x\right)$ for $j=1, \ldots, J$. Specifically, for $j=1, \ldots, J$, we use

$$
\begin{aligned}
& \pi_{J}\left(x \mid \boldsymbol{\xi}_{J}\right) \propto \prod_{j=1}^{J}\left(m x-q\left(\xi_{j}\right) y_{j}^{*}\right) \mathbb{1}(A x \leqslant b, x \geqslant 0), \\
& \pi_{J}\left(\xi_{j} \mid x\right) \propto\left(m x-q\left(\xi_{j}\right) y_{j}^{*}\right) p\left(\xi_{j}\right) .
\end{aligned}
$$

By starting at initial values $\left(x^{(0)}, \boldsymbol{\xi}_{J}^{(0)}\right)$ and sampling through the conditionals, we define a Markov chain where $\left(x^{(g)}, \boldsymbol{\xi}_{J}^{(g)}\right) \stackrel{D}{\longrightarrow}\left(x^{(\infty)}, \boldsymbol{\xi}_{J}^{(\infty)}\right)$ as $g \rightarrow \infty$, with the pair $\left(x^{(\infty)}, \boldsymbol{\xi}_{J}^{(\infty)}\right)$ having distribution $\pi_{J}\left(x, \boldsymbol{\xi}_{J}\right)$, where $\stackrel{D}{\longrightarrow}$ represents convergence in distribution. Then the marginal draws $x^{(g)}$ will have distribution $\pi_{J}(x)$. Hence, we can use the Monte Carlo average of our draws to estimate the mean of $\pi_{I}(x)$, which in turn provides us with an estimate of $x^{*}$. One of the advantages of jointly simulating decision variable $x$ and sources of uncertainty $\xi$ is that we take draws with high objective function values.

In many cases, the full conditional distributions may not be standard distributions, and in such cases, as 
proposed in Bielza et al. (1999), we use the Metropolis algorithm to draw samples. During the implementation of the Metropolis algorithm, the choice of the proposal distribution requires that we have an irreducible and aperiodic Markov chain. A standard choice is a symmetric distribution such as normal or heavy-tailed $t$ with appropriate scaling. Bielza et al. provides further discussion regarding the specification of the proposal distribution. The details of our algorithm can now be summarized as follows.

3.1.3. Algorithm 1. Here, the augmented probability space is constructed on $\left(x, \boldsymbol{\xi}_{J}\right)$. We assume that the optimal recourse action $y_{j}^{*}\left(x, \xi_{j}\right)$ for each $j$ th scenario is available (or can be obtained numerically) from the second stage as in the newsvendor problem.

Step 1. Start with initial values: $\left(x^{(0)}, \xi_{j}^{(0)}, j=1, \ldots, J\right)$, where $x^{(0)}$ satisfies the constraints $\left\{x \mid A x^{(0)} \leqslant b, x^{(0)} \geqslant 0\right\}$. Set $i=1$.

Step 2. Compute $y_{j}^{*}\left(x^{(i-1)}, \xi_{j}^{(i-1)}\right)$ for all $J$ scenarios.

Step 3. Let $u_{1}\left(x^{(i)}, \xi_{j}^{(i-1)}\right)=m x^{(i)}-q\left(\xi_{j}^{(i-1)}\right) y_{j}\left(x^{(i)}, \xi^{(i-1)}\right)$, and draw $x^{(i)}$ from the full conditional distribution

$$
\pi\left(x^{(i)} \mid \boldsymbol{\xi}_{J}^{(i-1)}\right) \propto \prod_{j=1}^{J} u_{1}\left(x^{(i)}, \xi_{j}^{(i-1)}\right) \mathbb{I}\left(A x^{(i)} \leqslant b, x^{(i)} \geqslant 0\right) .
$$

To draw $x^{(i)}$, generate a candidate $x_{*}^{(i)}$ from the proposal distribution $g_{1}\left(x_{*}^{(i)} \mid x^{(i-1)}\right)$ and evaluate $u_{1}\left(x_{*}^{(i)}, \xi_{j}^{(i-1)}\right)$.

Assuming $g_{1}$ is a symmetric proposal density, compute

$$
a_{1}=\min \left\{1, \prod_{j=1}^{J} \frac{u_{1}\left(x_{*}^{(i)}, \xi_{j}^{(i-1)}\right)}{u_{1}\left(x^{(i)}, \xi_{j}^{(i-1)}\right)}\right\}
$$

With probability $a_{1}$, set $x^{(i)}=x_{*}^{(i)}$; otherwise, keep $x^{(i)}=x^{(i-1)}$.

Step 4. Let $u_{2}\left(x^{(i)}, \xi_{j}^{(i)}\right)=m x^{(i)}-q\left(\xi_{j}^{(i)}\right) y_{j}^{*}\left(x^{(i)}, \xi_{j}^{(i)}\right)$. Draw $\xi_{j}^{(i)}$ for $j=1, \ldots, J$ from the full conditional distribution

$$
\pi\left(\xi_{j}^{(i)} \mid x^{(i)}\right) \propto u_{2}\left(x^{(i)}, \xi_{j}^{(i)}\right) p\left(\xi_{j}^{(i)}\right) .
$$

To draw $\xi_{j}^{(i)}$, generate a candidate $\xi_{j *}^{(i)}$ from the proposal distribution $g_{2}\left(\xi_{j *}^{(i)} \mid x^{(i)}\right)$ and evaluate $u_{2}\left(x^{(i)}, \xi_{j *}^{(i)}\right)$.

Assuming $g_{2}$ is a symmetric proposal density, compute

$$
a_{2}=\min \left\{1, \frac{u_{2}\left(x^{(i)}, \xi_{j *}^{(i)}\right)}{u_{2}\left(x^{(i)}, \xi_{j}^{(i)}\right)}\right\} .
$$

With probability $a_{2}$, set $\xi_{j}^{(i)}=\xi_{j *}^{(i)}$; otherwise, keep $\xi_{j}^{(i)}=\xi_{j}^{(i-1)}$.

Step 5. Set $i=i+1$. Repeat Steps 2-4 until the Markov chain is judged to have practically reached convergence.

In implementation of the algorithm, we check for convergence at every so many iterations. When convergence is achieved, we calculate the mean (or mode) of $x$ and compute the optimality gap. If the mean of $x$ is feasible for all scenarios in the second stage and the optimality gap is smaller than the tolerance level, we stop and decide that this value is the estimate of the optimal decision. To evaluate the optimality gap, we use the MRP of Bayraksan and Morton (2009) that will be discussed in the next section. If the optimality gap is not smaller than the tolerance, then our strategy is to increase the value of $J$ and to rerun the algorithm as to be implemented in $\S 4$.

3.1.4. Algorithm 2. When the optimal recourse action $y^{*}(x, \xi)$ is not available in closed form, the augmented probability model can be written as

$$
\begin{array}{r}
\pi_{J}\left(x, \boldsymbol{\xi}_{J}, \mathbf{y}_{J}\right) \propto \prod_{j=1}^{J}\left[\left(m x-q\left(\xi_{j}\right) y_{j}\right) p\left(\xi_{j}\right) \mathbb{I}(A x \leqslant b, x \geqslant 0,\right. \\
\left.\left.T x+W y_{j} \geqslant h, y_{j} \geqslant 0\right)\right],
\end{array}
$$

where $\mathbf{y}_{J}=\left(y_{1}, \ldots, y_{J}\right)$.

We need to draw samples from $\pi_{J}\left(x, \boldsymbol{\xi}_{J}, \mathbf{y}_{J}\right)$ using the Gibbs sampler, and in turn, this requires samples from the conditional distribution $\pi\left(\mathbf{y}_{J} \mid x, \boldsymbol{\xi}_{J}\right)$. Draws from this joint conditional distribution can be obtained from the independent conditionals $\pi\left(y_{j} \mid x, \xi_{j}\right)$ for $j=1, \ldots, J$. We can recognize that $y_{j}^{*}=y\left(x, \xi_{j}\right)$ is the optimal solution of the minimization problem at the second stage under scenario $\xi_{j}$. Specifically, $y_{j}^{*}$ is the minimum of the problem $\min _{y} q\left(\xi_{j}\right) y_{j}$ subject to $\left(T x+W y_{j} \geqslant h, y_{j} \geqslant 0\right)$, and following Pincus (1968), we can use the conditional distribution of $y_{j}$ as

$$
\begin{array}{r}
\pi\left(y_{j} \mid x, \xi_{j}\right) \propto \exp \left\{-J q\left(\xi_{j}\right) y_{j}\right\} \mathbb{\Perp}\left(T x+W y_{j} \geqslant h, y_{j} \geqslant 0\right), \\
\text { for } j=1, \ldots, J .
\end{array}
$$

Algorithm 1 then needs to be modified to incorporate drawing samples from the conditional distribution of $y_{j}$ 's. The modified algorithm is discussed in the appendix, and an implementation is presented. 


\subsection{Related Literature}

Our approach for finding the first-stage solution $x$ and maximizing $u(x)=E_{\xi}[u(x, \xi)]$ without resorting to gradient methods is related to the simulated annealing algorithm. Pure simulated annealing also powers up a function to be maximized in order to find its optimum (Kirkpatrick et al. 1983). Aarts and Korst (1988) provide a detailed discussion about the use of simulated annealing for optimization. Pincus (1968) proposes a solution for deterministic optimization problems by analytically computing the ratio of annealed integrals. However, as noted by Müller et al. (2004), standard simulated annealing algorithms (see van Laarhoven and Aarts 1987 for a review) assume availability of $u(x)$ for direct evaluation.

Pincus (1970) presents the use of the Metropolis algorithm to approximate the ratio of integrals. Similarly, APS evaluates $u(x)$ in an iterative manner that simultaneously addresses maximization and integration by using MCMC. When the utility (objective) function $u(x)$ can be evaluated exactly, the APS chain reduces to a standard simulated annealing algorithm as described by Müller (1999). Bielza et al. (1999) utilize APS to solve decision problems and describe the procedure while fixing $J=1$. Müller et al. (2004) extend it to the solution of decision problems where the utility function may not be evaluated exactly and might have complex, highdimensional decision spaces by using higher values of the augmentation parameter, J. Similar ideas have been utilized to solve maximization of expected utility problem (Jacquier et al. 2010), to compute maximum likelihood estimates in latent state variable models (Jacquier et al. 2007), and to solve stochastic dynamic programs (Polson and Sorensen 2011).

Our first algorithm extends the proposed APS approach to stochastic programs, particularly to twostage stochastic programs with recourse within constrained domains, which is our main difference from the approach of Müller et al. (2004). We also provide performance comparisons with sample average approximation method that is widely used in stochastic programming literature. In addition to that, Algorithm 2 (see the appendix) utilizes the deterministic optimization approach of Pincus (1968) to provide a distributional form of the second-stage decision in a two-stage sequential decision setting. In doing that, our paper provides a connection between Pincus (1968) and the
APS algorithm of Bielza et al. (1999). The latter did not consider constrained domains, and the former dealt with only one-stage problems where the objective function was not analytically available.

\subsection{APS Formulation of the Newsvendor Problem}

In this section, we consider the two-stage stochastic programming formulation of the newsvendor problem presented in §1.1. More specifically, we have

$$
\max _{x}\left\{-c x+E_{\xi}[Q(x, \xi)]\right\} \quad \text { such that } x \geq 0,
$$

where $\boldsymbol{\xi}=\left(\xi_{1}, \xi_{2}\right)$, and $Q(x, \boldsymbol{\xi})=s\left(\xi_{2}\right) y_{1}^{*}(x, \boldsymbol{\xi})+r y_{2}^{*}(x, \boldsymbol{\xi})$. The optimal recourse actions are given by $y_{1}^{*}(x, \xi)=$ $\min \left(d\left(\xi_{1}\right), x\right), y_{2}^{*}(x, \xi)=\max \left(x-d\left(\xi_{1}\right), 0\right)$ for $s\left(\xi_{2}\right) \geqslant r$, and $y_{1}^{*}(x, \boldsymbol{\xi})=0, y_{2}^{*}(x, \xi)=x$ for $s\left(\xi_{2}\right) \leqslant r$.

Following our development in the above, using $J$ iid copies of the random vector $\left(\xi_{1}, \xi_{2}\right)$, we can write the augmented distribution, $\pi_{J}\left(x, \boldsymbol{\xi}_{1 J}, \boldsymbol{\xi}_{2 J}\right)$, as

$$
\pi_{J}\left(x, \boldsymbol{\xi}_{1 J}, \boldsymbol{\xi}_{2 J}\right) \propto \prod_{j=1}^{J}\left(-c x+s\left(\xi_{2 j}\right) y_{1 j}^{*}+r y_{2 j}^{*}\right) p\left(\xi_{1 j}, \xi_{2 j}\right),
$$

where $\xi_{i, j}$ is the $j$ th draw of $\xi_{i}, \boldsymbol{\xi}_{i j}=\left\{\xi_{i, 1}, \ldots, \xi_{i, J}\right\}$ for $i=1,2, \boldsymbol{\xi}_{j}=\left\{\xi_{1 j}, \xi_{2 j}\right\}, y_{1 j}^{*}=y_{1}^{*}\left(x, \xi_{j}\right)$, and $y_{2 j}^{*}=y_{2}^{*}\left(x, \boldsymbol{\xi}_{j}\right)$.

We use Gibbs sampling and draw from the full conditional distributions, $\pi_{J}\left(x \mid \boldsymbol{\xi}_{1 J}, \xi_{2 J}\right)$ and $\pi_{J}\left(\xi_{1 j}, \xi_{2 j} \mid x\right)$ for $j=1, \ldots, J$. If $\xi_{1 j}$ and $\xi_{2 j}$ are independent, we can sample from their marginal conditionals. However, for any choice of the $p\left(\xi_{1 j}, \xi_{2 j}\right)$, one cannot directly draw samples from these distributions. We use a Metropolis algorithm as presented in the Algorithm 1, using Gaussian proposal distributions in both cases.

In $\S 4$ we assume the functions $d\left(\xi_{1 j}\right)=c_{1} \exp \left(\xi_{1 j}\right)$ and $s\left(\xi_{2 j}\right)=c_{2} \exp \left(\xi_{2 j}\right)$. In this case it can be shown that the full conditionals of $\xi_{1 j}$ and $\xi_{2 j}$ are both mixtures that still require the use of the Metropolis method.

As pointed out by Parpas et al. (2013), samples from multimodal distributions often preclude the application of stochastic programming. Our realizations of the random variable being dependent on the incumbent candidate solution and the use of $J$ realizations in each iteration make our algorithm a viable candidate for dealing with multimodal distributions. In $\S 4$ we consider the newsvendor problem with a bimodal demand distribution. More specifically, we assume that $d\left(\xi_{1}\right)^{\prime}$ s distribution is a mixture of two gamma densities. 


\section{Performance of the APS Algorithm}

In this section, we evaluate performance of APS algorithm using the newsvendor problem and compare it with the SAA algorithm. The SAA algorithm is chosen because it was adopted as the benchmark in related influential works (Higle and Sen 1996) and has been widely used to solve similar stochastic programs (Birge and Louveaux 2011). Evaluation of optimality gap plays an important role in our assessment of the algorithm's performance. To do this, we use the MRP of Mak et al. (1999) and Bayraksan and Morton (2009) to assess the quality of the candidate solution. In what follows, we give an overview of the MRP.

\subsection{Assessment of the Optimality Gap}

To assess the optimality, the optimal objective function value is either computed using the optimal solution $x^{*}$ or estimated using a Monte Carlo average (as in SAA) when $x^{*}$ is not analytically available. The optimality gap of a candidate solution, $x_{*}$, is found using $n_{g}$ replications with sample size $n$ in each case. A summary of the MRP procedure is provided below:

- For every replication, $k=1,2, \ldots, n_{g}$,

-Sample $n$ iid observations $\xi^{k}=\left\{\xi^{k 1}, \xi^{k 2}, \ldots, \xi^{k n}\right\}$ from $p(\xi)$.

- Solve the stochastic program for the sample of $\xi^{k}$ to compute $x_{k}^{*}$, an estimate for the optimal solution $x^{*}$.

-Compute the optimal objective function value for the sample $\xi^{k}$ as $Q\left(x_{k}^{*}, \xi^{k}\right)$.

-Compute the objective function value for the candidate solution $x_{*}$ for the sample $\xi^{k}$ as $Q\left(x_{*}, \xi^{k}\right)$.

-Compute the gap estimate, $D_{n}^{k}\left(x_{*}\right)=(1 / n)$. $\sum_{j=1}^{n}\left[\left|Q\left(x_{*}, \xi^{k j}\right)-Q\left(x_{k}^{*}, \xi^{k j}\right)\right|\right]$.

- Compute the average and sample variance of the gap estimate as

$$
\begin{aligned}
\bar{D}_{n_{g}}\left(x_{*}\right) & =\frac{\sum_{k=1}^{n_{g}} D_{n}^{k}\left(x_{*}\right)}{n_{g}}, \text { and } \\
s_{n_{g}}^{2}\left(x_{*}\right) & =\frac{1}{n_{g}-1} \sum_{k=1}^{n_{g}}\left(D_{n}^{k}\left(x_{*}\right)-\bar{D}_{n_{g}}\left(x_{*}\right)\right)^{2} .
\end{aligned}
$$

For large values of $n_{g}$, because of the central limit theorem, the confidence interval for the optimality gap can also be obtained.

\subsection{Performance Results}

In this section, we present the solution of the newsvendor problem with unimodal and bimodal demand distributions using the APS algorithm with different augmentation parameter values $J$ and the SAA algorithm. We present evidence of the effectiveness of APS versus SAA for both cases.

We first consider the unimodal case with $d\left(\xi_{1}\right)=$ $c_{1} \exp \left(\xi_{1}\right)$ and $s\left(\xi_{2}\right)=c_{2} \exp \left(\xi_{2}\right)$ as the demand and sales price functions, respectively. As defined before, the random vector $\xi=\left\{\xi_{1}, \xi_{2}\right\}$ follows a normal distribution that results in demand and sales price functions having lognormal distributions. We assume that $c_{1}=100, c_{2}=$ 1.5 , and $\xi_{1}$ and $\xi_{2}$ are independent normally distributed random variables with $\mu_{i}=0$ and $\sigma_{i}^{2}=1$ for $i=1,2$. Furthermore, the unit cost and the salvage values are set as $c=1$ and $r=0.1$, respectively. In this particular case, the actual optimal solution $x^{*}$ can be computed numerically.

The APS is run with $J$ values of 25,50 , and 100 . The SAA is run for 25 replications. Candidate solutions are computed every 100 iterations. We draw a sample of $\boldsymbol{\xi}$ of size 150,000 to estimate the continuous distribution, $p(\boldsymbol{\xi})$. The optimality gap is computed using mean absolute percentage error (MAPE). It is a function of the difference between the objective function value for the candidate solution of a particular approach and the objective function value for the optimal solution; both are computed using the same sample with 150,000 realizations.

Figure 2 presents numerical evidence of geometric convergence by providing optimality gaps for $J$ values of $\{10,25,50,100\}$ and cases of $10,000,20,000,30,000$, and 40,000 realizations where the number of realizations is given by $J \times G$, where $G$ is the number of MCMC iterations in each case.

In Figure 3, we present the MAPE for both SAA and APS algorithms. The results are presented for increments of 5,000 realizations for the SAA and for the APS with $J=25$ and $J=50$. For the case of APS with $J=100$, we present results for increments of 10,000 realizations. For example, in the case of APS with $J=$ 100 , a sample of 10,000 implies $G=100$ MCMC draws. It is important to note that Figure 3 does not show the MAPE for the APS with $J=25$ and $J=50$ for 10,000 realizations. This is due to the lack of convergence of the MCMC algorithm with $G=400$ draws for $J=25$ and $G=200$ draws for $J=50$. For 20,000 realizations, the MCMC chains are observed to have practically reached convergence using evidence of BGR values 
Figure 3 (Color online) Optimality Gap for SAA and APS with the Same Unimodal Demand Sample

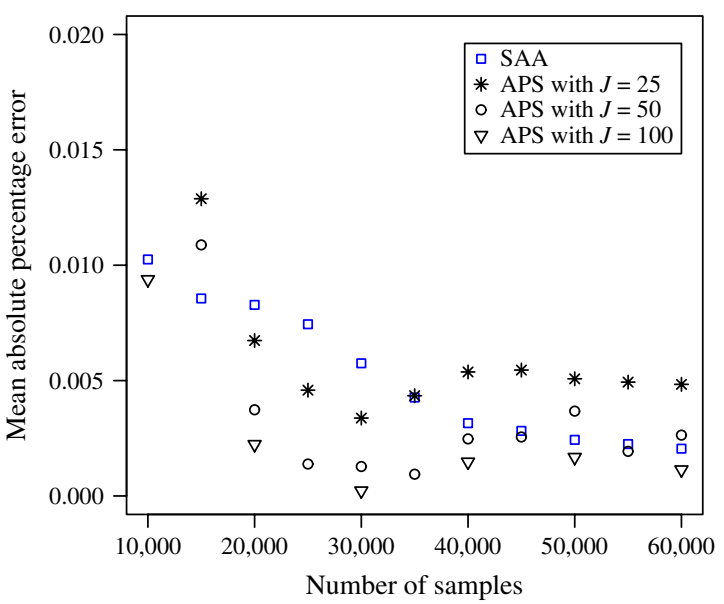

of $1.020,1.021$, and 1.027 for simulations with $J=25$, $J=50$, and $J=100$, respectively. We note that the APS with $J=100$ performs better than the SAA algorithm at any sample size. Even the APS with $J=25$ becomes superior to the SAA algorithm with 20,000 realizations. The SAA recovers and performs as well as or better than the APS with $J=25$ or $J=50$ for a larger number of realizations, but it does not perform as well as the APS algorithm with $J=100$.

We provide the box plots of optimality gaps for SAA and APS with $J=25$ and $J=100$ for 20,000, $30,000,40,000$, and 50,000 realizations (see Figure 4) to understand the evolution of the performance and provide further comparisons. Box plots of APS with $J=25$ do not exhibit any significant performance increase in the mean optimality gap but results show a reduced variance for increased sample sizes compared with the SAA. However, APS with $J=100$ provides smaller mean optimality gaps as well as smaller standard deviation values for any sample size.

Figure 5 elaborates on the visualization of the performance comparisons for a given sample size by presenting the box plots of the optimality gaps for all the approaches with 30,000 realizations. It can be seen that APS runs result in better mean absolute percentage optimality gaps, and their standard deviations are also smaller. Among the APS runs, those with larger values of $J$ provide better performance. Figure 6 provides the box plots of the first-stage decision variable $x$ compared with the optimal solution $x^{*}$. The standard deviation of the draws for a higher value of $J$ is smaller, which presents evidence for faster convergence.

Overall, these practical simulation runs are in line with our expectations from the theoretical results. The APS algorithm converges faster and performs consistently better than the SAA algorithm for large values of $J$. Thus, as an implementation strategy, once the MCMC convergence is achieved, it is better to increase $J$ rather than the number of MCMC iterations $G$ to improve the optimality gap of the APS algorithm.

Figure 4 Box Plots of the Optimality Gap with the Same Unimodal Demand Samples
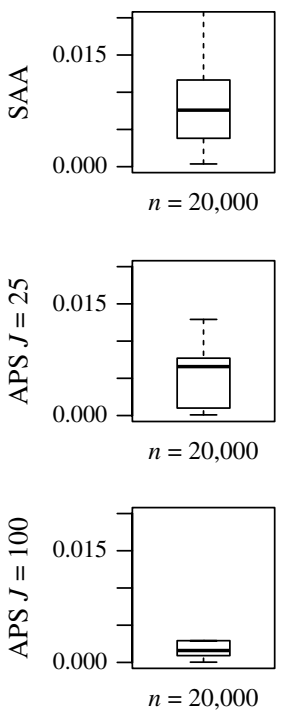
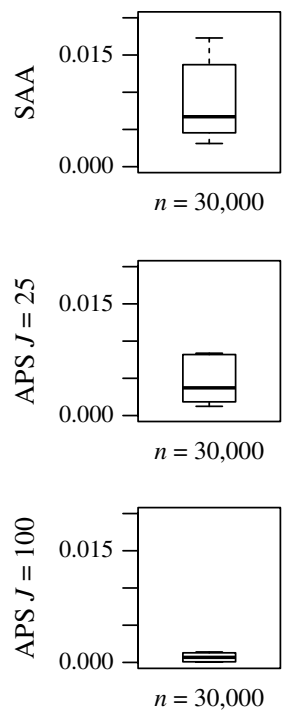
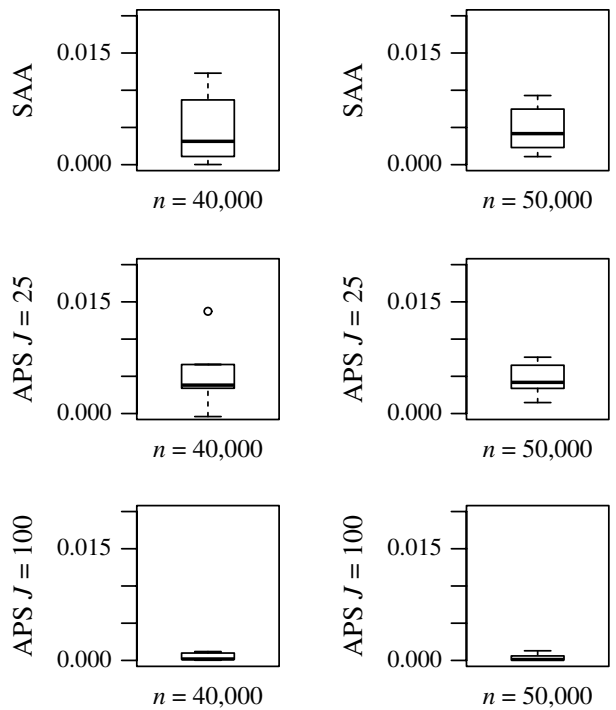
Figure 5 Box Plots of the Optimality Gap for SAA and APS with the Same Unimodal Demand Sample of 30,000 Draws

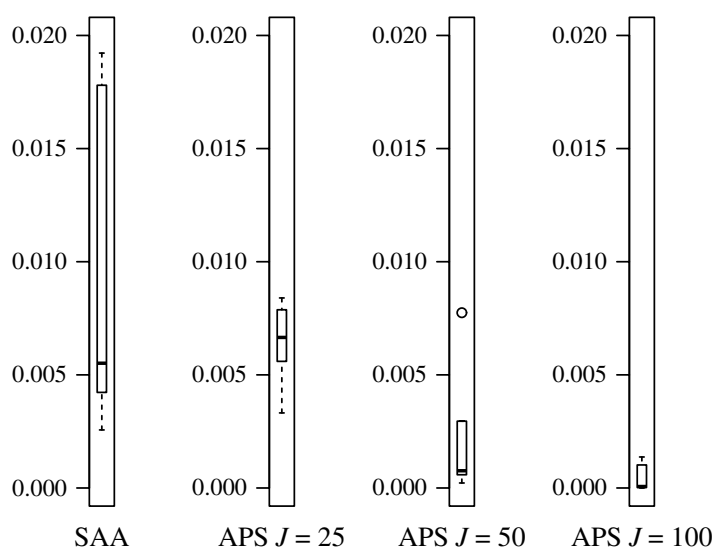

Another analysis is done to compare the samples of the random vector $\boldsymbol{\xi}$ drawn by SAA and APS methods. An expected advantage of the APS approach is smarter sampling from the state space, which leads to better objective function values. In Figure 7, we present the comparison of SAA and the APS with $J=25$. Similar results can be obtained for other values of $J$. We have used the samples of $\boldsymbol{\xi}$ that are drawn from each particular algorithm rather than the common independent sample. Objective function values are computed for the candidate solutions using the own samples of each algorithm and compared to the optimal objective function value that is computed for the exact optimal solution based on a large sample of 150,000 realizations. As we can see from Figure 7, the standard

Figure 6 (Color online) Box Plots of the First-Stage Decision $x$ for $J=25, J=50$, and $J=100$ with the Same Unimodal Demand Sample
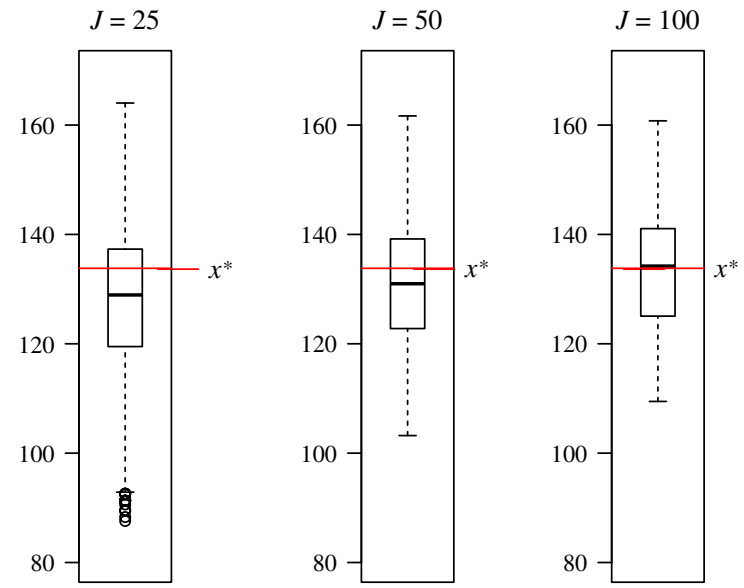

Figure 7 (Color online) Standard Deviation and Optimality Gap for SAA and APS with Individual Unimodal Demand Samples
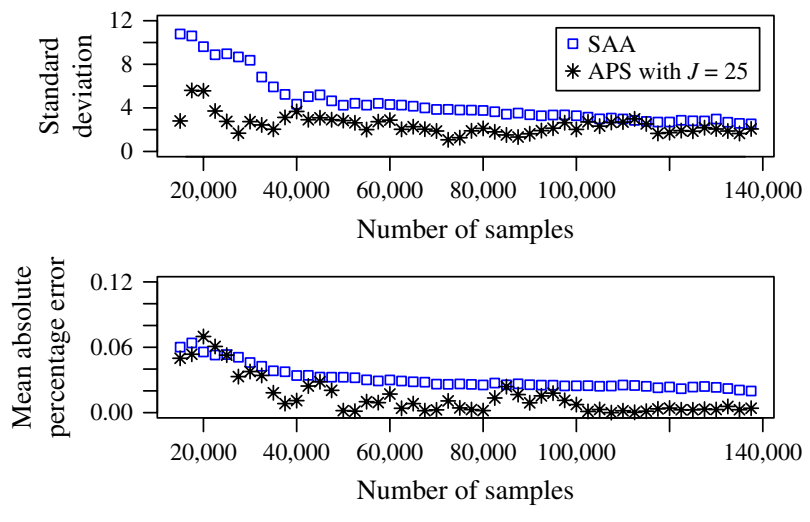

deviations of the objective functions are smaller for APS samples, which is in line with the importance sampling results of Parpas et al. (2013) and Infanger (1993). Furthermore, based on the MAPE, the optimality gap for APS approach is also consistently found to be smaller compared with the SAA approach.

Next, we consider the newsvendor problem with a bimodal demand distribution. Specifically, we assume that the distribution of $d\left(\xi_{1}\right)$ is a mixture of gamma densities as

$$
\begin{aligned}
p\left(d\left(\xi_{1}\right)\right)= & 0.5 \cdot \operatorname{Gamma}(a=150, b=1) \\
& +0.5 \cdot \operatorname{Gamma}(a=150, b=3),
\end{aligned}
$$

where $\operatorname{Gamma}(a, b)$ denotes a gamma density with a shape parameter $a$ and scale parameter $b$. We have $s\left(\xi_{2}\right)=c_{2} \exp \left(\xi_{2}\right)$ with a $c_{2}$ value of 1.5 , and $\xi_{2}$ is a normally distributed random variable with mean $\mu_{2}=0$ and variance $\sigma_{2}^{2}=1$.

In this case, the optimal solution cannot be computed easily via numerical methods. Thus, we have found the optimal solution and optimal objective function value using sample average approximation methods for a large enough sample of 150,000 realizations. This is used to estimate the optimal solution of the original problem.

The APS algorithm was implemented with $J$ values of 25,50 , and 100 . The SAA algorithm was run for 25 replications. We will not provide a detailed analysis and figures to keep the paper parsimonious. The MAPEs for the optimality gaps are reported in Figure 8. As can be clearly seen from the figure, APS with $J$ 
Figure 8 (Color online) Optimality Gap for SAA and APS with the Same Unimodal Demand Sample

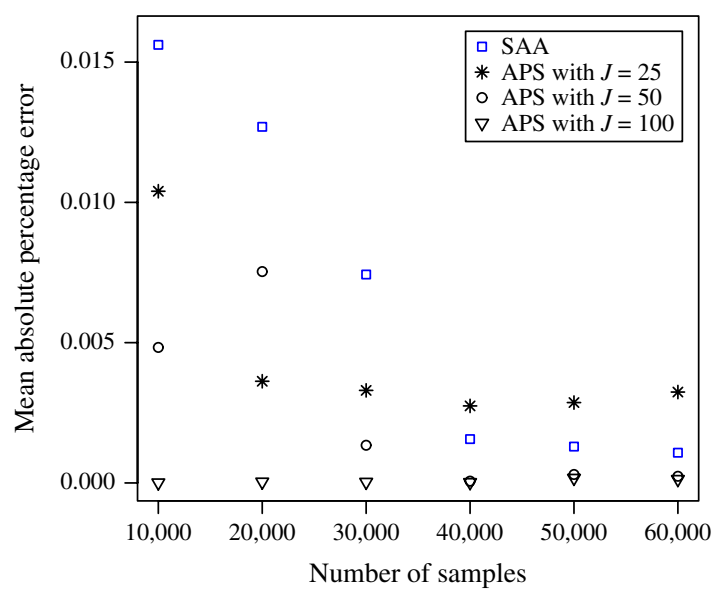

values of 50 and 100 is found to be superior for all number of samples. The box plot of the first-stage decision variable $x$ for the first 20,000 iterations also provides similar insights (see Figure 9). Increasing the $J$ values results in a smaller error from the optimal solution and a smaller standard deviation.

Similar to the analysis with unimodal demand, APS samples are found to be superior than the SAA Monte Carlo samples, as shown in Figure 10. APS provides objective function values with smaller standard deviations and smaller optimality gaps for all sample sizes. Overall, the efficiency of APS can be concluded

Figure 9 (Color online) Box Plots of the First-Stage Decision $x$ for $J=25, J=50$, and $J=100$ with the Same Unimodal Demand Sample
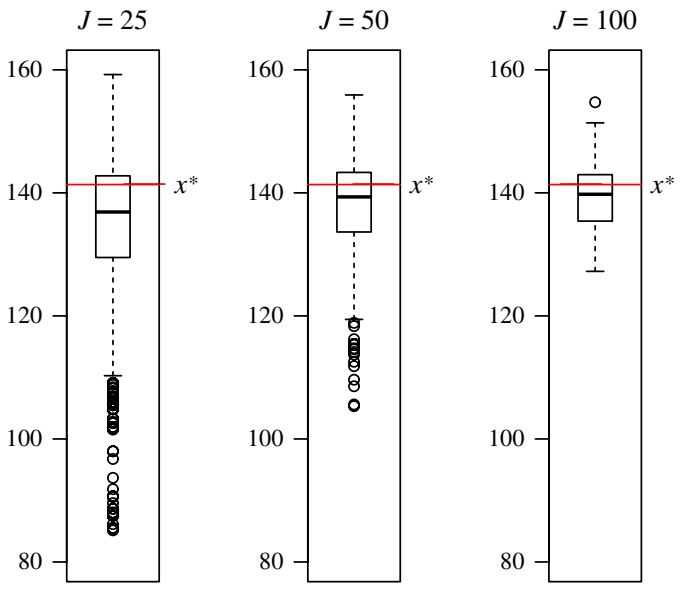

Figure 10 (Color online) Standard Deviation and Optimality Gap for SAA and APS with Individual Samples of Bimodal Demand
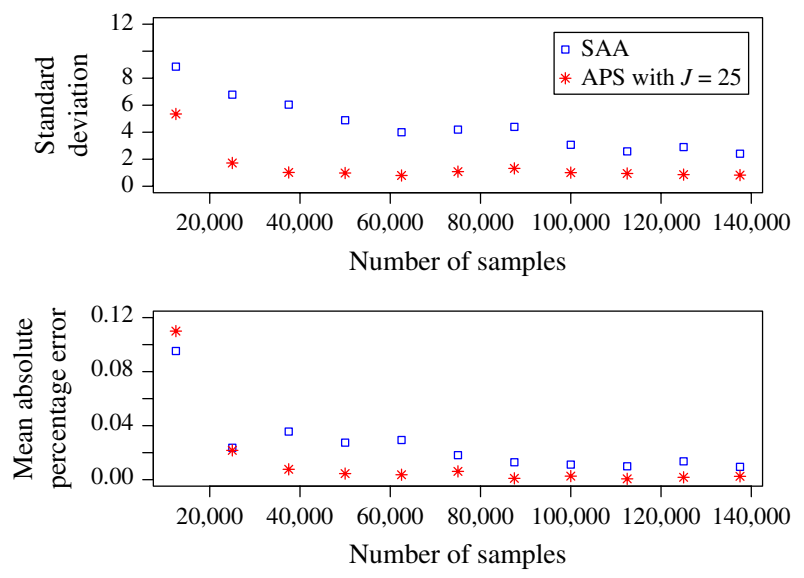

to increase especially when used with its own samples and large enough $J$ values.

The performance results are generally robust with respect to increasing the standard deviation of the random variables. As expected, for major increases of the standard deviation, both our algorithm and the SAA algorithm result in higher optimality gaps and variances when run with the same number of samples.

\section{Discussion}

Solving two-stage stochastic programming with recourse requires optimization and calculation of the expected recourse function, $Q(x)$. In this paper, simulation from the proposed augmented probability model, where both decision variables and uncertainty shocks are treated as stochastic, allows us to perform both simultaneously. In doing so, we sample more frequently from the augmented decision and state space in that the objective function has higher values. This avoids inefficient samples that otherwise would have been drawn without considering the optimization perspective. We also avoid having to use gradient-based approaches.

For performance comparison, the newsvendor problem is formulated as a two-stage stochastic program with recourse and solved by both the APS and SAA algorithms. It is shown that APS provides smaller optimality gaps for the same number of samples compared with SAA. APS also results in samples with reduced variance. In an APS algorithm, faster convergence can be achieved by increasing $J$. 
Markov chain Monte Carlo methods are used to sample from resulting nonstandard distributions and compute the optimal solution. We use the Metropolis algorithm to sample from the complete conditionals. However, sampling may not be straightforward, and the convergence may not be fast enough for some specific cases. When the objective function is flat and the optimal decision is not significantly better than its alternatives, the mode of the pseudo-distribution may not be distinct. Increasing the value of the augmentation parameter $J$ resolves this problem, since the mean converges to the optimal solution for high values of $J$.

During the implementation of the algorithm, a user may need to deal with certain issues. First, the objective function is assumed to be positive within the APS algorithm. For negative objective functions, certain transformations can be used to achieve nonnegativity (Jacquier et al. 2010). Second, sampling from constrained domains with an MCMC algorithm remains a challenge. The use of Lagrangian penalty parameters can be considered.

There are a number of directions for future research. The proposed approach can be extended to solve other multistage stochastic problems. It may be worthwhile to explore how it applies to various nonlinear stochastic programming problems and dynamic stochastic programs. Another interesting area for work is recourse problems where probability distribution of source of uncertainty is dependent on the first-stage decision variable; see, for example, Jonsbråten et al. (1998).

\section{Acknowledgments}

The authors thank the editors and two anonymous reviewers whose comments helped improve and clarify this manuscript.

\section{Appendix. Algorithm 2}

In cases where the optimal recourse action is not easy to evaluate, the Algorithm 1 of $\$ 3.1$ needs to be modified. In this case, the augmented probability space is constructed on $\left(x, \boldsymbol{\xi}_{J}, \mathbf{y}_{J}\right)$, and the full conditional distribution of $y_{j}$ 's can be constructed using the Pincus (1968) result. The details of our algorithm can be summarized as follows.

Step 1. Start with initial values: $\left(x^{(0)}, \xi_{j}^{(0)}, y_{j}^{(0)}, j=1\right.$, $\ldots, J)$, where $x^{(0)} \in S=\left\{A x^{(0)} \leqslant b, x^{(0)} \geqslant 0, y_{j}^{(0)} \geqslant 0, T x^{(0)}+\right.$ $\left.\left.W y_{j}^{(0)} \geqslant h\right)\right\}$. Set $i=1$.

Step 2. Let $u_{1}\left(x^{(i)}, \xi_{j}^{(i-1)}, y_{j}^{(i-1)}\right)=m x^{(i)}-q\left(\xi_{j}^{(i-1)}\right) y_{j}^{(i-1)}$ and draw $x^{(i)}$ from the full conditional distribution

$$
\begin{aligned}
\pi\left(x^{(i)} \mid \boldsymbol{\xi}_{J}^{(i-1)}, \mathbf{y}_{J}^{(i-1)}\right) \propto & \prod_{j=1}^{J}\left(u_{1}\left(x^{(i)}, \xi_{j}^{(i-1)}, y_{j}^{(i-1)}\right)\right. \\
& \left.\cdot \mathbb{I}\left(A x^{(i)} \leqslant b, x^{(i)} \geqslant 0\right)\right) .
\end{aligned}
$$

While using MCMC, generate a candidate $x_{*}^{(i)} \sim g_{1}\left(x_{*}^{(i)} \mid x^{(i-1)}\right)$ and evaluate $u_{1}\left(x_{*}^{(i)}, \xi_{j}^{(i-1)}, y_{j}^{(i-1)}\right)$, where $g_{1}$ is a symmetric proposal density. Compute

$$
a_{1}=\min \left\{1, \prod_{j=1}^{J} \frac{u_{1}\left(x_{*}^{(i)}, \xi_{j}^{(i-1)}, y_{j}^{(i-1)}\right)}{u_{1}\left(x^{(i)}, \xi_{j}^{(i-1)}, y_{j}^{(i-1)}\right)}\right\}
$$

With probability $a_{1}$, set $x^{(i)}=x_{*}^{(i)}$; otherwise, keep $x^{(i)}=x^{(i-1)}$.

Step 3. Let $u_{2}\left(x^{(i)}, \xi_{j}^{(i)}, y_{j}^{(i-1)}\right)=m x^{(i)}-q\left(\xi_{j}^{(i)}\right) y_{j}^{(i-1)}$. Draw $\xi_{j}^{(i)}$ for $j=1,2, \ldots, J$ from the full conditional distribution

$$
\pi\left(\xi_{j}^{(i)} \mid x^{(i)}, y_{j}^{(i-1)}\right) \propto\left(u_{2}\left(x^{(i)}, \xi_{j}^{(i)}, y_{j}^{(i-1)}\right)\right) p\left(\xi_{j}^{(i)}\right) .
$$

In so doing, generate a candidate $\xi_{j *}^{(i)} \sim g_{2}\left(\xi_{j *}^{(i)} \mid x^{(i)}, y_{j}^{(i-1)}\right.$ ) from the symmetric proposal density $g_{2}$ and evaluate $u_{2}\left(x^{(i)}, \xi_{j *}^{(i)}, y_{j}^{(i-1)}\right)$. Compute

$$
a_{2}=\min \left\{1, \frac{u_{2}\left(x^{(i)}, \xi_{j *}^{(i)}, y_{j}^{(i-1)}\right)}{u_{2}\left(x^{(i)}, \xi_{j}^{(i)}, y_{j}^{(i-1)}\right)}\right\} .
$$

With probability $a_{2}$, set $\xi_{j}^{(i)}=\xi_{j *}^{(i)}$; otherwise, keep $\xi_{j}^{(i)}=\xi_{j}^{(i-1)}$.

Step 4. Let $u_{3}\left(x^{(i)}, \xi_{j}^{(i)}, y_{j}^{(i)}\right)=m x^{(i)}-q\left(\xi_{j}^{(i)}\right) y_{j}^{(i)}$. Draw $y_{j}^{(i)}$ for $j=1,2, \ldots, J$ from the full conditional distribution

$$
\pi\left(y_{j}^{(i)} \mid x^{(i)}, \xi_{j}^{(i)}\right) \propto \exp \left\{-J q\left(\xi_{j}^{(i)}\right)\right\} \mathbb{I}\left(T x^{(i)}+W y_{j}^{(i)} \geqslant h\right) .
$$

In so doing, generate a candidate $y_{j *}^{(i)} \sim g_{3}\left(y_{j *}^{(i)} \mid x^{(i)}, \xi_{j}^{(i)}\right)$ and evaluate $u_{3}\left(x^{(i)}, \xi_{j}^{(i)}, y_{j *}^{(i)}\right)$. Assuming $g_{3}$ is a symmetric proposal density, compute

$$
a_{3}=\min \left\{1, \frac{u_{3}\left(x^{(i)}, \xi_{j}^{(i)}, y_{j *}^{(i)}\right)}{u_{3}\left(x^{(i)}, \xi_{j}^{(i)}, y_{j}^{(i)}\right)}\right\} .
$$

With probability $a_{3}$, set $y_{j}^{(i)}=y_{j *}^{(i)}$; otherwise, keep $y_{j}^{(i)}=y_{i}^{(i-1)}$.

Step 5. Set $i=i+1$. Repeat Steps $2-4$ until the Markov chain is judged to have practically converged.

In what follows we illustrate an implementation of Algorithm 2. Consider the following simple two-stage stochastic program:

$$
\max _{x}\{3 x-E[Q(x, \xi)]\}
$$

subject to $x \leqslant 3, x \geqslant 0$,

$$
\text { where } Q(x, \xi)=\min _{y} \xi y
$$

subject to $0.5 x+y \geqslant 5, y \geqslant 0$,

where $\xi$ has a lognormal distribution with parameters $\mu=0$ and $\sigma^{2}=0.1$. It is easy to see that the optimal solution for the first-stage problem is $x^{*}=3$. The optimal value for the decision variable is 3 , no matter the number of scenarios sampled, whereas the objective function value can change 
Figure A.1 (Color online) Trace Plots for the Decision Variable $x$ for Different Values of $J$

Trace plot of the decision variable $x$

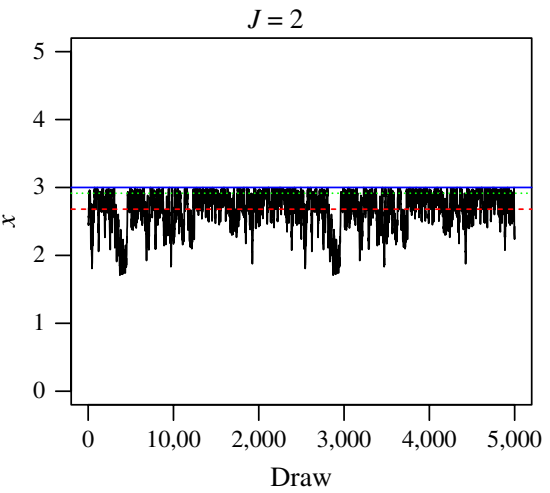

Trace plot of the decision variable $x$

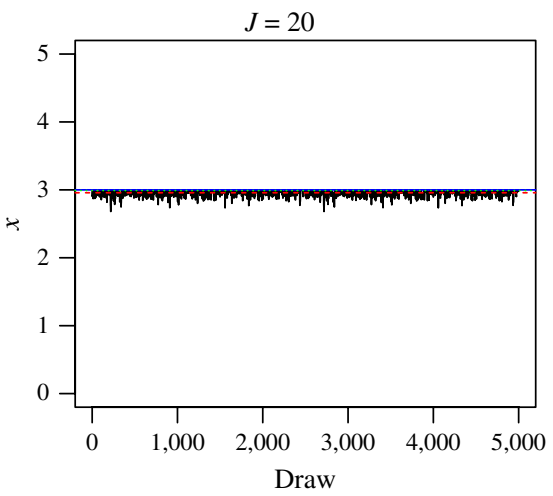

with the realizations of the random variable. Our augmented probability distribution is given by

$$
\begin{aligned}
\pi_{J}\left(x, \boldsymbol{\xi}_{J}, \mathbf{y}_{J}\right) \propto \prod_{j=1}^{J}\left(3 x-\xi_{j} y_{j}\right) p\left(\xi_{j}\right) \\
\quad \cdot \mathbb{I}\left(0 \leqslant x \leqslant 3, y_{j} \geqslant 5-0.5 x, y_{j} \geqslant 0\right) .
\end{aligned}
$$

In implementing Algorithm 2, we use a Gibbs sampler where the conditional distributions are given by

$$
\begin{aligned}
& \pi\left(x \mid \boldsymbol{\xi}_{J}, \mathbf{y}_{J}\right) \propto \prod_{j=1}^{J}\left(3 x-\xi_{j} y_{j}\right) p\left(\xi_{j}\right) \mathbb{I}(0 \leqslant x \leqslant 3), \\
& \pi\left(\xi_{j} \mid x, y_{j}\right) \propto\left(3 x-\xi_{j} y_{j}\right) p\left(\xi_{j}\right), \\
& \pi\left(y_{j} \mid x, \xi_{j}\right) \propto \exp \left\{-J \xi_{j} y_{j}\right\} \mathbb{I}\left(y_{j} \geqslant \max (0,5-0.5 x)\right),
\end{aligned}
$$

for $j=1, \ldots, J$, where we use the Pincus (1968) result to obtain the conditional distribution for $\xi_{j}$. We use our algorithm and the Metropolis method with normal proposal distributions to draw from the above distributions.

We run 5,000 iterations of our Gibbs sampler after an initial 500 burn-in samples. In Figure A.1, we present the trace plots from the simulations of decision variable $x$ for values

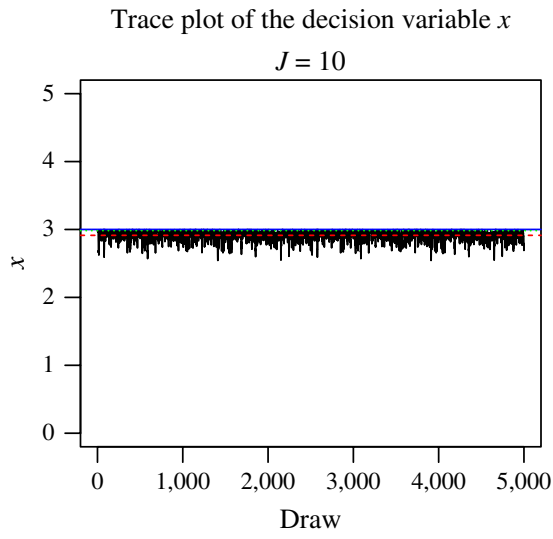

Trace plot of the decision variable $x$

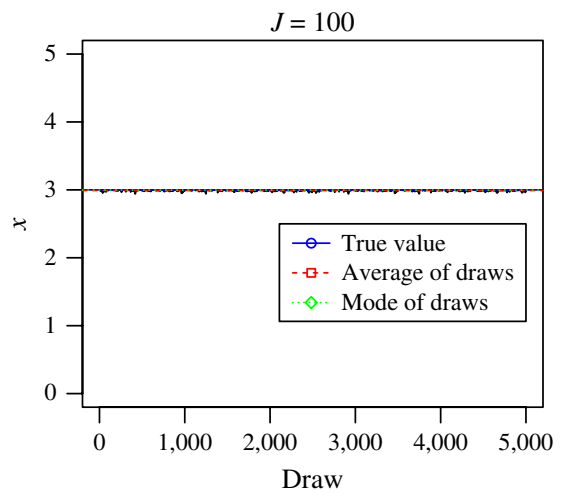

$J=2,10,20$, and 100 . The plots show that the algorithm converges to the true value of $x$ as $J$ increases. For $J=20$, we estimate the true value of the first-stage decision variable to be 2.998 , with a Monte Carlo error of $0.06 \%$.

\section{References}

Aarts E, Korst J (1988) Simulated Annealing and Boltzmann Machines: A Stochastic Approach to Combinatorial Optimization and Neural Computing (John Wiley \& Sons, New York).

Bayraksan G, Morton DP (2009) Assessing solution quality in stochastic programs via sampling. Oskoorouchi MR, ed. Tutorials in Operations Research (INFORMS, Hanover, MD), 102-122.

Benders JF (1962) Partitioning procedures for solving mixed-variables programming problems. Numer. Math. 4:238-252.

Bielza C, Müller P, Insua DR (1999) Decision analysis by augmented probability simulation. Management Sci. 45(7):995-1007.

Birge JR, Louveaux FV (1988) A multicut algorithm for two stage stochastic linear programs. Eur. J. Oper. Res. 34(3):384-392.

Birge JR, Louveaux F (2011) Introduction to Stochastic Programming (Springer-Verlag, Berlin).

Brooks SP, Roberts GO (1998) Assessing convergence of Markov chain Monte Carlo algorithms. Statist. Comput. 8(4):319-335.

Dai L, Chen CH, Birge JR (2000) Convergence properties of two-stage stochastic programming. J. Optim. Theory Appl. 106(3):489-509. 
Dantzig GB (1955) Linear programming under uncertainty. Management Sci. 1(3-4):197-206.

Dantzig GB, Thapa MN (1997) Linear Programming 2: Theory and Extensions (Springer-Verlag, New York).

Dempster AP, Laird NM, Rubin DB (1977) Maximum likelihood from incomplete data via the EM algorithm. J. Roy. Statist. Soc. Ser. B 339(1):1-38.

Ermoliev Y (1988) Stochastic quasigradient methods. Ermoliev Y, Wets RJ-B, eds. Numerical Techniques for Stochastic Optimization (Springer-Verlag, Berlin), 141-185.

Gaivoronski A (1988) Stochastic quasigradient methods and their implementation. Ermoliev Y, Wets RJ-B, eds. Numerical Techniques for Stochastic Optimization (Springer-Verlag, Berlin), 313-351.

Gelman A, Rubin DB (1992) Inference from iterative simulation using multiple sequences. Statist. Sci. 7(4):457-472.

Geman D, Geman SA (1987) Relaxation and annealing with constraints. Complex Systems Technical Report 35, Division of Applied Mathematics, Brown University, Providence, RI.

Higle JL, Sen S (1991) Stochastic decomposition: An algorithm for two-stage linear programs with recourse. Math. Oper. Res. 16(3):650-669.

Higle JL, Sen S (1996) Stochastic Decomposition: A Statistical Method for Large Scale Stochastic Linear Programming (Kluwer Academic Publishers, Norwell, MA).

Homem-de-Mello T, Bayraksan G (2014) Monte Carlo sampling-based methods for stochastic optimization. Working paper, Universidad Adolfo Ibanez, Santiago, Chile. http://www.optimization -online.org/DB_FILE/2013/06/3920.pdf.

Infanger G (1993) Monte Carlo (importance) sampling within a Benders decomposition algorithm for stochastic linear programs. Ann. Oper. Res. 39(1):69-95.

Isaacson DL, Madsen RW (1976) Markov Chains, Theory and Applications, Vol. 4 (John Wiley \& Sons, New York).

Jacquier E, Johannes M, Polson N (2007) MCMC maximum likelihood for latent state models. J. Econometrics 137(2):615-640.

Jacquier E, Johannes M, Polson N (2010) Maximum expected utility via MCMC. Working paper, Centre for Interuniversity Research and Analysis on Organizations, HEC Montréal, Montréal. http://faculty.chicagobooth.edu/nicholas.polson/ research/papers/mcmceu.pdf.

Jonsbråten TW, Wets RJ-B, Woodruff DL (1998) A class of stochastic programs with decision dependent random elements. Ann. Oper. Res. 82:83-106.

Kirkpatrick S, Gelatt CD Jr, Vecchi MP (1983) Optimization by simulated annealing. Science 220(4598):671-680.

Mak WK, Morton DP, Wood RK (1999) Monte Carlo bounding techniques for determining solution quality in stochastic programs. Oper. Res. Lett. 24(1-2):47-56.

Müller P (1999) Simulation-based optimal design. Bernardo JM, Berger JO, Dawid AP, Smith AFM, eds. Bayesian Statistics 6 (Oxford University Press, Oxford, UK), 459-474.

Müller P, Sansó B, De Iorio M (2004) Optimal Bayesian design by inhomogeneous Markov chain simulation. J. Amer. Statist. Assoc. 99(467):788-798.

Parpas P, Ustun B, Webster M, Tran QK (2013) Importance sampling in stochastic programming: A Markov chain Monte Carlo approach. Working paper, Imperial College London, London. http://www.optimization-online.org/DB_FILE/2012/08/ 3558.pdf.

Pennanen T, Koivu M (2005) Epi-convergent discretizations of stochastic programs via integration quadratures. Numer. Math. 100(1):141-163.
Pincus M (1968) A closed form solution of certain programming problems. Oper. Res. 16(3):690-694.

Pincus M (1970) A Monte Carlo method for the approximate solution of certain types of constrained optimization problems. Oper. Res. 18(6):1225-1228.

Polson NG, Sorensen M (2011) A simulation-based approach to stochastic dynamic programming. Appl. Stochastic Models Bus. Indust. 27(2):151-163.

Rubinstein RY, Shapiro A (1993) Discrete Event Systems: Sensitivity Analysis and Stochastic Optimization by the Score Function Method, Vol. 346 (John Wiley \& Sons, New York).

Shapiro A (2003) Monte Carlo sampling methods. Ruszczyński A, Shapiro A, eds. Stochastic Programming, Handbooks in Operations Research and Management Science, Vol. 10 (Elsevier, Amsterdam), 353-425.

Shapiro A, Homem-de-Mello T (1998) A simulation-based approach to two-stage stochastic programming with recourse. Math. Programming 81(3):301-325.

Shapiro A, Homem-de-Mello T (2000) On the rate of convergence of optimal solutions of Monte Carlo approximations of stochastic programs. SIAM J. Optim. 11(1):70-86.

Shapiro A, Dentcheva D, Ruszczyński AP (2009) Lectures on Stochastic Programming: Modeling and Theory (Society for Industrial and Applied Mathematics, Philadelphia).

Spall JC (2005) Introduction to Stochastic Search and Optimization: Estimation, Simulation, and Control (John Wiley \& Sons, Hoboken, NJ).

Tierney L (1994) Markov chains for exploring posterior distributions. Ann. Statist. 22(4):1701-1728.

van Laarhoven PJ, Aarts EHL (1987) Simulated Annealing: Theory and Applications (Reidel, Amsterdam).

Van Slyke RM, Wets R (1969) L-shaped linear programming with application to optimal control and stochastic programming. SIAM J. Appl. Math. 17(4):638-663.

Tahir Ekin is assistant professor of quantitative methods at McCoy College of Business, Texas State University. He holds a Ph.D. in decision sciences from the George Washington University and a B.S. in industrial engineering from Bilkent University, Turkey. He conducts research in the areas of simulation-based stochastic programming, stochastic modeling, and Bayesian inference, including applications to medical fraud detection.

Nicholas G. Polson is Robert Law, Jr. professor of econometrics and statistics at the University of Chicago, Booth School of Business. He is a Bayesian statistician who conducts research on financial econometrics, Markov chain Monte Carlo, particle learning, and Bayesian inference. His article "Bayesian analysis of stochastic volatility models" was named one of the most influential articles in the 20th anniversary issue of the Journal of Business and Economic Statistics.

Refik Soyer is chair and professor of decision sciences and statistics at the George Washington University (GWU). He is the Mitch Blaser Distinguished Scholar in Business Analytics and also serves as the director of the Institute for Integrating Statistics in Decision Sciences at GWU. He received his D.Sc. in operations research from GWU, M.Sc. in operational research from the University of Sussex, United Kingdom, and B.A. in economics from Boğaziçi University, Turkey. 